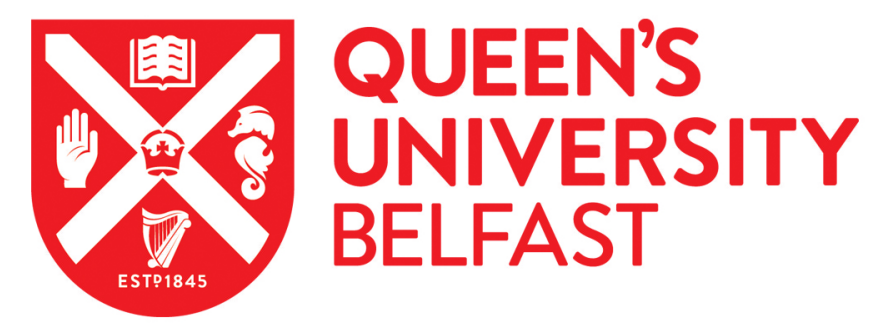

\title{
Facilitators' delivery of a psychosocial intervention in a controlled trial for men with prostate cancer and their partners: a process evaluation
}

\author{
Parahoo, K., McKenna, S., Prue, G., McSorley, O., \& McCaughan, E. (2017). Facilitators' delivery of a \\ psychosocial intervention in a controlled trial for men with prostate cancer and their partners: a process \\ evaluation. Journal of Advanced Nursing. https://doi.org/10.1111/jan.13248
}

Published in:

Journal of Advanced Nursing

Document Version:

Peer reviewed version

Queen's University Belfast - Research Portal:

Link to publication record in Queen's University Belfast Research Portal

Publisher rights

(c) 2016 John Wiley \& Sons Ltd. This work is made available online in accordance with the publisher's policies. Please refer to any applicable terms of use of the publisher.

\section{General rights}

Copyright for the publications made accessible via the Queen's University Belfast Research Portal is retained by the author(s) and / or other copyright owners and it is a condition of accessing these publications that users recognise and abide by the legal requirements associated with these rights.

Take down policy

The Research Portal is Queen's institutional repository that provides access to Queen's research output. Every effort has been made to ensure that content in the Research Portal does not infringe any person's rights, or applicable UK laws. If you discover content in the Research Portal that you believe breaches copyright or violates any law, please contact openaccess@qub.ac.uk. 
Title:

Facilitators' delivery of a psychosocial intervention in a controlled trial for men with prostate cancer and their partners: a process evaluation

Running head: Facilitators' delivery of a psychosocial intervention.

Authors:

Kader Parahoo* BA PhD RMN

Professor in Nursing and Health Research

Institute of Nursing and Health Research, Ulster University, Cromore Road, Coleraine, BT521SA,

Northern Ireland.

ak.parahoo@ulster.ac.uk

Suzanne McKenna BSc MSc PhD

Research Associate

Institute of Nursing and Health Research, Ulster University, Shore Road, Newtownabbey, Co.Antrim, BT570QB, Northern Ireland.

smt.mckenna@ulster.ac.uk

Gillian Prue BSc PhD

Lecturer in Chronic illness

School of Nursing and Midwifery, Queen's University Belfast, Medical Biology Centre, 97 Lisburn Rd, Belfast BT9 7BL, Northern Ireland

g.prue@qub.ac.uk

Oonagh McSorley BSc RN

Project Manager

Northern Ireland Cancer Registry, Queen's University Belfast, Mulhouse Building, Grosvenor Rd, BT12 6DP Northern Ireland.

o.mcsorley@qub.ac.uk

Eilis McCaughan BSc PhD RN

Professor in Cancer Care

Institute of Nursing and Health Research, Ulster University, Cromore Road, Coleraine, BT521SA,

Northern Ireland.

em.mccaughan@ulster.ac.uk

*Contact details for the corresponding author: as above

\section{Acknowledgements}

To all those who facilitated the intervention and to Cancer Focus Northern Ireland, who provided

funding for the project.

There is no conflict of interest.

All authors have agreed on the final version and meet at least one of the following criteria (recommended by the ICMJE*):

1) substantial contributions to conception and design, acquisition of data, or analysis and interpretation of data;

2) drafting the article or revising it critically for important intellectual content. 


\section{Abstract}

Aim. The aim of this paper is to report the process evaluation of facilitators' delivery of a psychosocial intervention (called CONNECT), in a randomised controlled trial, to men with prostate cancer and their partners.

Background: There is a lack of information on the process of implementing psychosocial interventions in controlled trials and, in particular, on the role and performance of facilitators who deliver them. Yet this information is crucial in assessing whether these interventions are effective or not and why.

Methods: Semi-structured qualitative interviews and diaries were used to collect data (January - October 2012) from four facilitators and a co-facilitator. Data were analysed using the Miles et al. framework.

\section{Results:}

Five themes were discernible. These were 'difficulties to keep to the structure of the intervention', 'selective coverage of topics', 'partner participation', 'overall impression of the group and telephone sessions' and 'perceived benefits to participants'. Issues such as not keeping to the aim of the intervention, deviating from the content and/or reluctance in discussing sensitive issues such as sexual health may mean that the psychosocial effects of the intervention may not have been fully realised.

Conclusions: These findings will be useful for further development and evaluation of the intervention. A tentative conceptual framework of factors, related to facilitators, influencing the fidelity of interventions in the context of controlled trials, is offered. This model, which requires further development and testing, will be useful for researchers worldwide who are involved in developing interventions and training facilitators. 
(245 words)

Key words: qualitative, controlled trials, psychosocial intervention, facilitators, fidelity, process evaluation, nurses

\section{Why is this research or review needed?}

- Although psychosocial interventions are increasingly being offered to cancer patients and survivors, their effectiveness is yet to be demonstrated.

- The need for process evaluation, which has the potential to reveal why an intervention works or not, is well recognised.

- There is, however, a lack of information on how facilitators deliver these interventions, in the context of a controlled trial.

\section{What are the key findings?}

- This study shows that, despite involving facilitators in the development of an intervention and training them, the intervention may not be delivered as planned.

- There are several factors including facilitators' background, values, attitudes and competence that can influence intervention delivery.

- A conceptual model of factors, relating to facilitators, which can influence intervention delivery in the context of a controlled trial, is offered. 


\section{How should the findings be used to influence research?}

- Clinicians and researchers can use this model to inform how they train, support and supervise facilitators and monitor their performance.

- Researchers should pay more heed to the central role that facilitators play in trials of the effectiveness of psychosocial interventions. 


\section{Introduction}

A cancer diagnosis and the treatment that follows can have significant physical and psychosocial effects on cancer sufferers and these effects can last well into remission and beyond (Parahoo et al, 2013). Some of these effects include emotional distress, anxiety, uncertainty and depression, which in turn can affect their quality of life. Psychosocial interventions in cancer, which have a history dating back to the 1970s (Klemm et al., 2003) have been developed worldwide to help and support people with cancer and their carers to cope with some of these effects. As the number of these interventions increases, it is important for clinicians, including nurses, to know if they are effective or not. Systematic reviews have not shown the magnitude of their effectiveness that practitioners and researchers expected to see (Parahoo et al., 2013). The reasons put forward to explain the discrepancy between researchers' high expectations and the results of controlled trials include: data collection tool not being sensitive enough to detect subtle changes in participants' wellbeing, including quality of life (Vilhauer, 2010) and inappropriate use of theoretical frameworks to underpin the intervention (Hulbert-Williams et al., 2014). There has been less focus, however, on whether the interventions were delivered by facilitators as planned.

The challenges of maintaining research protocol fidelity in drug trials are well documented (see e.g. Lawton et al., 2011). Compared with drug or surgical interventions, psychosocial interventions are relatively more difficult to implement in a randomised controlled trial. This is why psychosocial interventions have been described as complex interventions (Craig et al, 2008). These psychosocial interventions may look easy, on paper, to implement but may present difficulties when implemented in the real world of practice. The role of facilitators 
(defined as lay or professional persons whose task it is to implement interventions) is crucial as to whether the intervention is delivered as planned.

Nurses play a key role in the development and the delivery of these psychosocial interventions (see e.g. Northouse et al., 2007; Mishel et al., 2009). In a systematic review studies of psychosocial interventions for men with prostate cancer, Parahoo et al. (2013) reported that nurses delivered or were involved in delivering the interventions in eight out of the 19 included studies.

\section{Background}

There seems to be lack of information on the process of implementing psychosocial interventions, in particular the role of facilitators, in reports of randomised controlled trials. Randomised controlled trials of these types of interventions have been described as the 'black box' since it can be difficult to know why the intervention worked (or not) without exploring the underlying processes (Grant et al., 2013). Researchers have not focussed specifically on facilitators and how they actually implement the intervention (Hoey et al., 2011). Often, there are only one or two facilitators in a trial of interventions and this may be considered too few to merit a study. It is clear from the literature that facilitators are at the frontline of psychosocial care for cancer survivors (Owen et al., 2009) and that studying what facilitators do, can provide researchers 'with important insights into how their theoretically and empirically guided interventions 'work' when implemented in real-world community and clinical settings' (Senn and Carey, 2012). Participants, too, have reported that facilitators played an important role in the process of change (Silvergleid and Mankowski, 2006) and 
that they perceived facilitators' input as having an influence on psychological outcomes and quality of life of group members (Lieberman and Golant, 2002).

Few relevant studies were identified after an extensive search of the literature. In a Canadian study, Owen et al. (2009) explored the benefits and challenges experienced by professional facilitators of online support groups for cancer survivors, using a qualitative approach. They reported that one of the most frequently mentioned issues was ‘facilitator role uncertainty'. Facilitators questioned how much they should interact and how directive this interaction should be with group members (Owen et al., 2009). In a qualitative study of the learning experience of six psycho-oncology counsellors who facilitated online support groups for cancer patients in the United States (US), Stephen et al. (2011) reported that 'co-facilitating' with an expert, debriefing and participating in peer supervision group, were critical to their role as facilitators. Hoey et al. (2009), in a study comparing the needs of health professional and peer cancer support group facilitators in Australia, reported that health professional facilitators were more likely to regard training and debriefing as valuable and beneficial, than peers.

There are more studies on facilitators of support groups (online and face-to face) than on those facilitating psychosocial interventions. Although one can learn from these studies, facilitating support groups is not the same as facilitating psychosocial interventions (McCaughan et al., 2015). The latter is aimed at changing behavior intentionally, while in the former, the facilitator mainly 'facilitates' the exchange of information and experience between group members.

Two studies (similar to the one reported here) that explored the perspectives of facilitators in delivering an intervention were identified. Senn and Carey (2012), in their US study, carried 
out focus groups and individual interviews with eight experienced facilitators who delivered safe sex interventions, as part of a randomized controlled trial. They reported that several facilitator characteristics, including being open and non-judgmental, being flexible and being culturally competent were important in ensuring successful implementation of interventions. Chujo and Okamura (2015) carried out a study, in Japan, of the skills of three facilitators in a psychosocial intervention trial for cancer patients, using a qualitative approach. They observed that both new and experienced facilitators were unable to 'perform skilful intervention’ and reported that facilitators ‘had difficulty keeping concentration’ when sessions were long (p.8). Chujo and Okamura (2015) concluded that these problems could have countered the aim of the intervention which was to improve participants' coping abilities and their quality of life.

Together, these studies highlight the different facets and concerns regarding facilitators of psychosocial interventions. The purpose of this paper is to add to the increasing knowledge regarding what facilitators experience and do by focusing on the first UK-based randomized controlled trial of a psychosocial intervention for men with prostate cancer and their partners. The lessons learnt will be useful to make appropriate changes to the intervention and to inform future trials of these types of interventions.

\section{The intervention}

This psychosocial intervention (called CONNECT), based on the FOCUS programme [Northouse et al., 2007] in the US, has been described in detail (McCaughan et al., 2013). It is a nine-week programme consisting of three two-hour group sessions (on weeks 1, 3 and 9) and two telephone sessions to men with prostate cancer and their partners (weeks 5 and 7). The overall aim of this facilitator-led intervention was to enhance the couple's (men and their partners) belief in their ability to manage their cancer and related issues. The main outcomes 
were self-efficacy, quality of life, uncertainty, symptom distress and healthy behaviours. The sessions consisted mainly of discussions on symptom management, sexual and urinary dysfunction, uncertainty management, positive thinking and healthy lifestyles (see appendices 1 and 2). The aim was to encourage couples to adopt healthy coping strategies. For example, each couple was asked to select one aspect of their lifestyle that they wanted to change. Examples included ‘doing more exercise’, such as starting a 15-minute daily walk or doing relaxation exercises once a day.

These sessions were supplemented by information sheets developed for the FOCUS project and adapted for use in Northern Ireland. Health care professionals, users and facilitators contributed to their development. The information sheets provided the triggers for group discussion and they were also available for participants to take home and read in their own time.

Although there were concrete themes to be addressed in the group sessions, there was a degree of fluidity to the intervention. During each group session there was a constant movement to and from each of the topics. The intervention was underpinned by the themes of self-empowerment and journey navigation. Each of the group sessions was designed with the aim of assisting couples to become effective self-managers and helping them to effectively navigate their way through the prostate cancer journey. The group sessions were conducted in a way that facilitated personal/couple development and achievement of goals that were relevant to the couple's needs.

A further opportunity to individualise the intervention occurred with the telephone sessions. The telephone sessions were considered booster sessions where content from the previous 
two group sessions was reinforced and very little new content was introduced. These sessions provided the opportunity for the intervention to be individualised as participants were encouraged to set their own personal targets.

\section{Evaluation model}

The Re-Aim framework (Glasgow et al., 1999) was used to evaluate the overall feasibility of implementing the intervention and the effects it had on participants. We used several data collection tools including standardised tools, interviews, diaries and observations. Some components including the experience and perceptions of participants, recruitment strategies and the cost of the intervention have already been reported in the literature (McCaughan et al., 2014; McCaughan et al., 2015). In this paper we report on the facilitators' delivery of the intervention.

The Re-Aim framework has been used in several studies to evaluate the implementation process of psychosocial interventions (Boersma et al., 2015). The framework comprises five components: Reach, Efficacy, Adoption, implementation and Maintenance. In this study we focus on the 'implementation' component of the framework to collect data on whether the intervention was delivered as intended. Implementation, at the setting level, refers to the intervention agents' fidelity to the various elements of an intervention's protocol, including consistency of delivery as intended. At the individual level, implementation refers to clients' use of the intervention strategies (Glasgow et al., 1999).

\footnotetext{
Aim

The aim of this paper is to report the process evaluation of facilitators' delivery of a psychosocial intervention (called CONNECT), in a randomised controlled trial to men with prostate cancer and their partners.
} 


\section{Design}

The Medical Research Council (Craig et al., 2008) recommends the use of qualitative methods to explore the perceptions of participants and personnel, as part of the process of the evaluation of complex interventions. The efficacy of the CONNECT intervention was evaluated by means of a trial (McCaughan et al., 2014). The experience and perception of couples participating in the interventions were explored through qualitative interviews and questionnaires (McCaughan et al., 2015). A descriptive, qualitative approach (Sandelowski, 2000) comprising multiple methods (described below) was used in this study to explore the facilitators' delivery of the intervention.

\section{Data collection}

Three qualitative methods were used to collect data from all four facilitators and the cofacilitator. This included one-off semi-structured qualitative interviews (each lasting just over one hour) with each individual (including the co-facilitator). All facilitators also kept a diary to record the topics covered during sessions, the amount of time spent on each, the duration of the whole session and their thoughts on the delivery of the intervention. The co-facilitator kept notes about what happened in the sessions regarding the fidelity of intervention delivery. The interviews (carried out at a university site) consisted of broad questions relating to their previous experience in group work, their views on the structure of the programme (including, timing, duration, frequency and dose), their experience of delivering the group and telephone sessions, the benefits of the programme for the men with prostate cancer and for their partners and their views on how the intervention could be improved. The interviews were digitally recorded (with the participants’ permission) and transcribed by SM. 
The purpose of participant observation (by the co-facilitator) was to report on the fidelity of the intervention but also to support the facilitator in delivering the intervention as intended.

\section{Participants}

Between January 2012 and October 2012, the intervention was delivered to four groups (of 34 dyads). Each group was delivered by a different facilitator $(n=4)$. Facilitators, who were recruited from a national cancer charity, had training and experience in counselling people with cancer generally, but not specifically with men with prostate cancer and their partners. Although they had experience in leading support groups and one-to-one sessions, they did not have prior experience of delivering a structured group (face-to-face) and telephone psychosocial intervention, such as CONNECT. One of the researchers [OM] co-facilitated all the group sessions as a participant observer. There is some evidence that co-facilitation is helpful in supporting group sessions (Cancer Council Australia, 2005). The facilitators were aware of the co-facilitator's role.

The four facilitators and the co-facilitator participated in the development of the programme and received a 5-day training course to familiarise themselves with the programme. Training was led by an expert who worked on a similar project in the US (Northouse et al., 2007). A protocol handbook was produced by the research team. Training content included, among other things, familiarisation with the CONNECT protocol, facilitators' role in the trial, the importance of adherence to rigour, maintaining fidelity of the intervention as well as information on prostate cancer, its physical, psychological and emotional impact and its treatment and side effects. Training was given on group facilitation, in particular how to manage group dynamics. Learning strategies included problem-solving, role play and discussion. 


\section{Ethical considerations}

Ethical approval was granted by the Office for Research Ethics in Northern Ireland. Informed consent from the facilitators and the co-facilitator was obtained prior to data collection. Assurances of confidentiality were given to all participants.

\section{Data analysis}

Data from all the diaries and interviews were handled through the N-Vivo software. Interview data were coded and analysed using Miles et al. Framework (Miles et al., 2013). Two researchers (SM and KP) carried out the analysis of all the data independently. These were then discussed with the other two researchers (EM and OM) with a view to resolving any conflicting interpretation and reaching a consensus.

The analysis process began during the data collection phase, when the researcher kept field notes. When the interview, diary and observation scripts and notes were transcribed, the large number of data were reduced by grouping them into manageable codes and categories (data reduction). This was followed by displaying the data by means of a 'spider diagram' to integrate codes from the interviews, diaries and observations (data display). The final stage involved merging codes into themes, interpreting, verifying and cross-referencing with field notes and transcripts (drawing conclusions).

\section{Rigour}

Lincoln and Guba’s (1985) four criteria for establishing trustworthiness: credibility, confirmability, dependability and transferability were used to establish rigour in this study. Credibility was ensured by the use of more than one method to study the same phenomenon. Confirmability was achieved both by methodological triangulation and confirmation of the findings by the participants. Adequate detail of the process of the study is reported in this 
paper for readers to assess the dependability of the findings. Additionally, data analysis and interpretation were carried out independently by two researchers and further discussed with the other two researchers. Although the primary aim of the study was to provide data on the feasibility of the CONNECT intervention, the findings will be of use to other clinicians and researchers involved in developing and implementing such interventions, thus ensuring some degree of transferability.

\section{Findings}

The findings from the analysis of diaries and interviews are presented together; where appropriate, quotes will be attributed to its source (e.g. facilitator interview [FI], facilitator diary [FD], co-facilitator diary [CD], co-facilitator interview [CI]). The numbers refer to particular facilitators.

Three of the four facilitators were female, as was the co-facilitator. They all had over five years' experience as counsellors except the co-facilitator who had an oncology nursing background. Five themes were prominent in the data. These were: 'difficulties to keep to the structure of the intervention'; 'selective coverage of topics’; ‘partner participation’; 'overall impression of the group and telephone sessions'; and 'perceived benefits to participants'.

\section{Difficulties keeping to the structure of the intervention}

All four facilitators found it challenging and felt under pressure delivering the programme mainly because, in the first session, participants wanted time to talk. As a result, facilitators were concerned that they would not have time to cover all of the issues they intended. They were used to conducting counselling sessions where the structure was 'fairly loose'. They found that there were a large number of topics to cover: 
It was challenging to give couples time to tell their story whilst keeping them on track without seeming to be dismissive of what they wanted to say. (FD1)

In terms of trying to go through the various areas, that was difficult. (FI4)

The co-facilitator reported that the extent to which facilitators followed the intervention as prescribed, varied greatly among facilitators. Some facilitators admitted that it was difficult 'not to go in counselling mode' and recognised that the CONNECT programme was different from counseling. This pressure to 'stick to the programme' was felt less in the next two sessions. The third and last group session was, according to the facilitators, more of a 'recapping session' with plenty of time to address requests identified in the telephone sessions. Two facilitators had so much time to spare in the last session that they carried out meditation exercises with participants (this was not part of the CONNECT programme). All facilitators reported in their diaries that co-facilitation was essential to the delivery of the intervention as the co-facilitator kept bringing them back to the programme.

\section{Selective coverage of topics}

Some topics (in the programme) were not covered because the facilitator did not see the need for them, in their particular groups. For instance bowel and rectal issues were not dealt with in detail because facilitators highlighted that participants had reported that they did not experience these problems or kept quiet about them. Facilitators in some sessions also reported that some topics such as communication or sexual dysfunction 'were not relevant' and explained that there was 'good communication' between the men and their partners: 
The couples had been married for a long time and they seemed, in the first session, to be expert in communicating with each other so I felt that it (sexual relationship) wasn’t an issue. (FD3)

All three couples indicated that sexual difficulties was not a particularly big issue for them as they were settled and happy. (FD2)

Some facilitators perceived that there was some reluctance on the part of the participants to talk about sexual issues. As one facilitator explained:

They didn't name it (sexual dysfunction) in the first session but in the second session we said, 'yes, something maybe is missing' and he (one male participant) said I know what you are going to say and named it himself. They did talk about it but I think they brushed over it.' . . . 'I think my group, just with the age groups maybe to a degree felt 'oh, we are in our late 60 s or 70 s now, that is not really a major issue'. But yes, I would agree it is an area that isn't brought up and I don't know whether that is we as professional as well. (FI3)

However, it appeared that there was also some reluctance on the facilitator's part to 'take on' this issue. According to the co-facilitator:

One couple wanted to discuss sexual dysfunction but this did not happen. I felt the facilitator was very uncomfortable with the subject. (CD)

\section{Partners’ participation}

All four facilitators reported that the men 'were quite vocal' and that it took time for the partners (all women) to fully participate in the groups: 
The wives gradually introduced themselves. I felt they seemed to sit back and think this is for the men here. (FI2)

The men tended to dominate the conversation and I had to encourage the wives to comment. They would have made the odd comment. (FD1)

The co-facilitator, who was present at all four group sessions, commented that when the men took over, the facilitators would 'bring them back with: 'how did the wives feel'?' She also reported that 'all the men, bar one, seemed to be putting on a front, saying 'I'm just getting on with things as usual'. According to the co-facilitator, they seemed to use humour to talk about their problems, while the women were more direct when they contributed to the discussion. Apart from one man, the women were the ones who took notes in these groups.

\section{Overall impression of the group and telephone sessions}

Overall, apart from the issues mentioned above, the facilitators were overwhelmingly supportive of the CONNECT programme. They reported that they were satisfied with the number, duration, timing and frequency of the sessions. Despite difficulties with 'keeping on track' with the programme, they acknowledged that having a structure to the sessions was very helpful. They also reported some difficulties with the telephone sessions.

The aims of the two telephone sessions were as follows: to monitor the couple's progress, check the targets they had set themselves and how they were achieving this target. The telephone sessions lasted, on average, between 15-30 minutes. Not all couples had speaker phones; therefore some conversations were with the men and their partner, separately. 
The first part of the telephone sessions seemed to be focused on how the participants were feeling. The men talked about their forthcoming appointments, their treatment and symptoms (e.g. blood pressure, urinary dysfunction). In contrast to the 'united front' which couples demonstrated in the group sessions, there were signs of tension and conflict in their relationships. As one facilitator explained:

I did not get the impression that the couple were in a position to work together to set goals or to manage symptoms together due to different information being shared by each of them. (FD4)

Another facilitator (FD3) felt that the partners would have liked to talk to her separately on her own. She wrote in her diary that one couple 'had a lot of issues between themselves' which she only 'discovered in the telephone session'. This was the same facilitator who earlier reported (in her diary) that the couples in her group session were 'expert communicators'.

Some physical and psychosocial issues, not identified or expressed in the group sessions, were 'brought up' in the telephone sessions. One man mentioned his 'dribbling problems' and another reported frequent trips to the toilet. Another couple who indicated (in the group sessions) that sexual difficulties 'was not a particularly big issue' for them as they were 'very settled and happy', talked (in the telephone session) about the 'sadness that this part of their relationship had gone'. 
According to all four facilitators, most of the couples had not set any short term targets. Those who did, reported broad targets such as 'managing weight', 'going for a walk', ‘booking a holiday', or 'walking the dog':

I asked couples to think of and identify one goal/target that they could work with...not sure if all the participants found this easy. (FI1)

Patient was unable to set a short-term action plan and to be specific. Talked about 'goals' in a more general way. (FD3)

Some facilitators recognised that the idea of couples setting targets 'did not work' and that it might have been 'due to my lack of being able to deliver that' (FI2\&3). Realising that target setting was a problem, one facilitator used the last group session to 'chat around the idea of target setting-what helps and what hinders’ (FI1).

\section{Perceived benefits to participants}

The overall impression from facilitators (gained by facilitators from the verbal feedback from participants at the end of the seminar) were that the groups sessions were very beneficial for the couples as it provided them with opportunities to hear the experiences of others, validate and share their own experiences with others:

There was a kind of validation in that 'I am not alone', or 'I'm not going crazy with this’....so validation.... Partners also learnt from other partners. (FI1)

The facilitators reported that some men mentioned that their 'frequency of urination had eased', others reported 'better communication within couples' and that 'things had changed for the better, openly discussing prostate cancer now'. All participants also found the 
information sheets, which provided a trigger to many of their discussion, very informative and helpful.

The co-facilitator summed up the benefits of the intervention:

I don't know whether it was just hearing other people's story, knowing they are not alone. I don't know if that's enough to make them go forward or whether it is in the information that they are given that they have got confidence that what they feel is normal. In terms of couple care, I'm not so sure because I felt they are ‘already there’. (CI)

The facilitators requested more flexibility in meeting the spontaneous, expressed needs of participants. Some wanted the third group session to be 'more open' to meet the needs identified in the first two group sessions. All recognised that the telephone sessions did not work as planned and that in terms of the focus on couples, it seemed that the women supported their partners and 'played down' their own issues. There was also an overwhelming feeling that four to five couples in each group session would be an ideal size. Overall they were more 'happy' with the intervention package and its delivery that they had anticipated.

\section{Discussion}

This evaluation identified several issues and problems that could have directly affected the fidelity of the intervention. These are: variations in the extent to which individual facilitators delivered the intervention as specified, the selective coverage of topics, the difficulties in implementing the behavioural components of the intervention (such as meeting behavioural 
targets and achieving them) and the introduction of exercises/activities which were not part of the programme.

Variations in the extent to which the facilitators followed the structure of the programme is a serious issue. It is acknowledged that implementing interventions in real life requires a degree of flexibility to address the needs of participants and to respond to unforeseen events. However, when the effectiveness of an intervention is being tested in a randomised controlled trial, it is important that facilitators keep as close to the programme as possible while maintaining an empathetic attitude towards participants (Senn and Carey, 2012).

There are questions, in this study, regarding the extent to which the essential components that make this particular intervention 'psychosocial' were delivered. Setting and achieving targets are central to a self-management programme. It seems that both participants and facilitators had difficulties in doing so. Behavioural simulations and role plays have been found to be particularly challenging to facilitators (Senn and Carey, 2012). From the facilitators' accounts, it appeared that participants benefited most from sharing experiences and getting information from the information sheets. One could ask whether a peer-support group with some input from health professional in providing information would have achieved the same effects as the CONNECT programme (the way it was delivered).

Prostate cancer and its treatment can lead to sexual dysfunction (Baniel et al., 2000). There are doubts, in this study, as the extent to which sexual dysfunction, as a topic, was adequately addressed by facilitators. Relying on appearances that couples seemed to communicate well with each other is not an effective way of assessing whether they have psychosexual problems or not. More serious is the assumption that some facilitators made regarding the 
link between 'stage of life' and sexual needs. Health professionals should avoid making 'assumptions about the possible meaning of the loss of sexual function for men of particular ages’ [O’Brien et al., 2011].

A recent national survey reported that 'many people remain sexually active in later life' and that 'sexual health is an important component of overall health and well-being' (Mitchell et al., 2013). There is also evidence that healthcare professionals 'routinely avoid discussing sexual problems' and that men, in particular, 'encounter difficulties in raising sexual issues during consultation' (Taylor and Gosney, 2011) and when they do, they adopt a stoic stance which reflect their masculinity (McCaughan et al., 2009).

There was unanimous agreement among facilitators that the two telephone sessions did not work as planned; it seemed that the aim of these sessions were not clear to facilitators or participants. However, they provided an opportunity for couples to talk about what was 'on their mind'. An un-anticipated bonus of the telephone calls was the 'discovery' that some participants had issues (such as urinary and sexual dysfunction) that they were reluctant to reveal in group sessions. Although telephone interventions have been found to be effective in addressing psychosocial needs, adequate training of facilitators is also required (Craven et al., 2013). One of the benefits of combining group and telephone sessions in this study is that it provided participants with different opportunities to express their needs and concerns.

There was some indication that wives in this study perceived that this intervention was for their partners. This is probably why they played a subsidiary role, focusing less on their needs, contributing less to the discussion and taking notes. Therefore the partners in this study may not have benefited as much as expected from this intervention. 


\section{Implications for practice}

In the first place, the findings of this study has implications for the CONNECT intervention. Although couples as well as facilitators expressed satisfaction with the intervention, it does not seem that the potential for behaviour change was fully maximised. Selective coverage of topics affects the fidelity of the intervention. Adding new 'activities' such as meditation exercises confounds the results. Therefore, facilitators should be made aware of the implications of being flexible to the extent that the integrity of the psychosocial intervention is compromised (Radziewicz et al., 2009). Facilitators need more training to deliver the psychosocial components of the programme to ensure that all the objectives of the intervention are achieved. In particular, they need to be confident in their ability to help participants disclose and address issues such as sexual dysfunction and couples’ relationship. The beliefs and attitudes of facilitators regarding sensitive issues should be assessed and appropriate training offered.

\section{Conceptual model}

This is the first reported study that has used a qualitative methodology to provide an insight into the issues and problems regarding the implementation of a psychosocial intervention for men with prostate cancer of their partners. Although the purpose of this study was to find out how feasible it would be for a larger study, these findings can be helpful to others contemplating the development, implementation and evaluation of a psychosocial intervention. To this end, a tentative conceptual model of factors, relating to facilitators, that can potentially influence the delivery of psychosocial interventions, in the context of a trial, is

offered (Figure 1). The evidence (from this study) that underpins this model is detailed in Table 1. Overall, these factors, if attended to, may help to ensure competent delivery of the 
intervention. It is acknowledged that due to the complexity of human interaction and the unpredictability of real life situations, flexibility and the ability to improvise are crucial. It is not expected that all facilitators in a project will deliver the intervention in exactly the same way. However, flexibility should not be at the expense of compromising the integrity of the trial. The fidelity of intervention delivery is important, if firm conclusions are to be drawn about the effectiveness of interventions. Facilitators play a key role in this process.

\section{Limitations of the study}

The main limitation of this study relates to the size of the sample. However, it is rare for this type of study, to have as many or more participants. Most trials of psychosocial interventions have one facilitator delivering the intervention (Parahoo et al., 2013) Another limitation relates to the fact that the interviewer was a member of the research team. This may have prevented them from being 'candid' about the intervention, as the interviewer led the development of the intervention. With hindsight, an interviewer outside the team should have carried out the interviews.

There is always the risk that the participant observer may have been selective in what to observe and report. Intensive debriefing helped the co-facilitator to reflect on how she interpreted the findings.

\section{Conclusions}

This study provides much needed insight into the crucial role that facilitators have in ensuring the effectiveness of complex interventions. No matter how good a psychosocial intervention is on paper, its effectiveness depends on how it is delivered. When delivery depends on multiple facilitators, the potential for variation and bias increases. Researchers should give as 
much attention to training, supporting and monitoring facilitators, as they do in developing psychosocial interventions.

The conceptual model of factors, relating to facilitators, which can influence the delivery of psychosocial interventions should be further developed and tested. It provides a framework that focuses attention on the crucial role of facilitators and their influence on the outcome of psychosocial interventions. This study fills an important gap in our understanding of what may actually happens during the implementation phase of a psychosocial intervention, in particular how those entrusted with its delivery, experience this process.

\section{$\underline{\text { Acknowledgements }}$}

To all those who facilitated the intervention and to Cancer Focus Northern Ireland, who provided funding for the project. 
References

Baniel J, Israilov S, Segenreich E, Livne PM (2000) Comparative evaluation of treatments for erectile dysfunction in patients with prostate cancer after radical retropubic prostatectomy. British Journal of Urology International 88, 58-62.

Boersma P, van Weert JC, Lakerveld J, Dröes RM (2015) The art of successful implementation of psychosocial interventions in residential dementia care: a systematic review of the literature based on the RE-AIM framework. International Psychogeriatrics 27,1:19-35.

Cancer Council Australia (2005)Building effective cancer support groups: report to the Department of Health and Ageing. The Cancer Council Australia 2005. (71 pages).

Chujo M, Okamura H (2015) The Skills of Facilitator Nurses in Psycho-Social Group Intervention for Cancer Patients. Yonago Acta medica 58, 69-75.

Craig P, Dieppe P, Macintyre S, Michie S, Nazareth I, Petticrew M (2008) Developing and evaluating complex interventions: the new Medical Research Council Guidance. British Medical Journal 337, a1655.

Craven O, Hughes CA, Burton A, Saunders MP, Molassiotis A (2013) Is a nurse-led telephone intervention a viable alternative to nurse-led home care and standard care for patients receiving oral capecitabine? Results from a large prospective audit in patients with colorectal cancer. European Journal of Cancer Care 22, 413-419. 
Glasgow RE, Vogt TM, Boles SM (1999) Evaluating the public health impact of health promotion interventions: the RE-AIM framework. American Journal of Public Health 89, $1322-27$.

Grant A, Treweek S, Dreischulte T, Foy R, Guthrie B (2013) Process evaluations for clusterrandomised trials of complex interventions: a proposed framework for design and reporting. Trials 14, 15.

Hoey LM, Sutherland G, Williams PA, White V (2011) Comparing the needs of health professional and peer cancer support group facilitators in an Australian context. European Journal of Cancer Care (Engl) 20, 87-92.

Hulbert-Williams,NJ, Storey L, Wilson, KG (2015), Psychological interventions for patients with cancer: psychological flexibility and the potential utility of Acceptance and Commitment Therapy. Eur J Cancer Care, 24: 15-27.

Klemm P, Bunnell D, Cullen M, Soneji R, Gibbons P, Holecek A (2003). Computers Informatics Nursing 03, 21, 136-42.

Lawton J, Jenkins N, Darbyshire JL, Holman RR, Farmer AJ , Hallowell N (2011) Challenges of maintaining research protocol fidelity in a clinical care setting: A qualitative study of the experiences and views of patients and staff participating in a randomized controlled trial. Trials, 12: 108. 
Lieberman M.A, Golant M (2002) Leader behaviors as perceived by cancer patients in professionally directed support groups and outcomes. Group Dynamics: Theory, Research and Practice 6, 267-276.

Lincoln, YS. \& Guba, EG. (1985). Naturalistic Inquiry. Newbury Park, CA: Sage Publications.

McCaughan E, Prue G, McSorley O, Northouse L, Schafenacker A, Parahoo K (2013) A randomized controlled trial of a self-management psychosocial intervention for men with prostate cancer and their partners: a study protocol. Journal of Advanced Nursing 69, 257283.

McCaughan E, McKenna S, McSorley O, Parahoo K (2015) The experience and perceptions of men with prostate cancer and their partners of the CONNECT psychosocial intervention: a qualitative exploration. Journal of Advanced Nursing 71, 8: 1871-82.

McCaughan E, Prue G, Parahoo K (2009) A systematic review of quantitative studies reporting selected patient experienced outcomes, with a specific focus on gender differences in people with colorectal cancer. European Journal of Oncology Nursing 13, 376-85.

McCaughan E, Parahoo K, McKenna S, McSorley O, Prue G, O’Sullivan J, Atkinson L (2014) Developing and evaluating a psychosocial intervention for men with prostate cancer and their partners. University of Ulster, Northern Ireland. 130 pages. 
McCaughan E, Parahoo K, Hueter I, Northouse L. Online support groups for women with breast cancer (Protocol). Cochrane Database of Systematic Reviews 2015, Issue 4. Art. No.: CD011652. DOI: 10.1002/14651858.CD011652.

Miles MB, Huberman AM, Saldaña J (2013) Qualitative Data Analysis: An Expanded Sourcebook (3rd Edition). Thousand Oaks, California. Sage.

Mishel MH, Germino BB, Lin L, Pruthi RS, Wallen EM, Crandell J, Blyler D (2009) Managing uncertainty about treatment decision making in early stage prostate cancer: a randomized controlled trial. Patient Education and Counseling 2009;77 (3):349-59.

Mitchell KR, Mercer CH, Ploubidis GB, Jones KG, Datta J, Field N, Copas AJ, Tanton C, Erens B, Sonnenberg P, Clifton S, Macdowall W, Phelps A, Johnson AM, Wellings K (2013) Sexual function in Britain: findings from the third National Survey of Sexual Attitudes and Lifestyles (Natsal-3). Lancet 382, 1817-29.

Northouse LL, Mood DW., Schafenacker A, Montie JE, Sandler HM, Forman JD, Hussain M, Pienta KJ, Smith DC, Kershaw T (2007) Randomized clinical trial of a family intervention for prostate cancer patients and their spouses. Cancer 110, 2809-2818.

O'Brien R, Rose P, Campbell C, Weller D, Neal RD, Wilkinson C, McIntosh H, Watson E (2011) Prostate Cancer Follow-up Group. 'I wish I'd told them': a qualitative study examining the unmet psychosexual needs of prostate cancer patients during follow-up after treatment. Patient Education and Counseling 84, 200-7. 
Owen JE, Bantum EO, Golant M (2009) Benefits and challenges experienced by professional facilitators of online support groups for cancer survivors. Psycho-Oncology 18, 144-55.

Parahoo K, McDonough S, McCaughan E, Noyes J, Semple C, Halstead EJ, Neuberger MM, Dahm P (2013) Psychosocial interventions for men with prostate cancer. Cochrane Database Systematic Review 12, CD008529.

Radziewicz RM, Rose JH, Bowman KF, Berila RA, O'Toole EE, Given B (2009) Establishing treatment fidelity in a coping and communication support telephone intervention for aging patients with advanced cancer and their family caregivers. Cancer Nursing 32, 193202.

Sandelowski M. (2000) Whatever happened to qualitative description. Research in Nursing and Health 23, 334-340.

Senn TE, Carey MP (2012) Voices from the trenches: facilitators' perspectives on HIV risk reduction interventions. Health Promotion Practice 13, 797-806.

Silvergleid CS, Mankowski ES (2006) How batterer intervention programs work: participant and facilitator accounts of processes of change. Journal of Interpersonal Violence 21, 139-59.

Stephen JE, Christie G, Flood K, Golant M, Rahn M, Rennie H, Speca M, Taylor-Brown J, Turner J (2011) Facilitating online support groups for cancer patients: the learning experience of psycho-oncology clinicians. Psycho-Oncology, 20, 8:832-40. 
Taylor A, Gosney MA (2011) Sexuality in older age: essential considerations for healthcare professionals. Age Ageing, 40,5:538-43.

Vilhauer RP, McClintock MK, Matthews AK (2010) Online support groups for women with metastatic breast cancer: a feasibility pilot study. Journal of Psychosocial Oncology, 28, 5:560-86. 
Table 1 Findings from this study to underpin the model

\begin{tabular}{|c|c|c|}
\hline actors & Findings & Comments \\
\hline $\begin{array}{l}\text { Previous experience } \\
\text { and training }\end{array}$ & $\begin{array}{l}\text { In this study all participants } \\
\text { had counselling experience } \\
\text { and training. Some found it } \\
\text { difficult to get out of the } \\
\text { 'counselling mode'. Some } \\
\text { introduced 'exercises' that } \\
\text { they have used previously but } \\
\text { which were not part of the } \\
\text { programme. } \\
\text { As counsellors, they were not } \\
\text { used to such behaviour } \\
\text { change programme. } \\
\text { Facilitating participants to set } \\
\text { and achieve objectives was } \\
\text { problematic. On the positive } \\
\text { side, their experience in } \\
\text { counselling people meant that } \\
\text { they were able to deal with } \\
\text { difficult situations as they } \\
\text { arose. }\end{array}$ & $\begin{array}{l}\text { Previous experience and training } \\
\text { can be both beneficial and a } \\
\text { hindrance. Facilitators can bring a } \\
\text { wealth of experience as health } \\
\text { professionals but could also be set in } \\
\text { their ways of practice. On the other } \\
\text { hand, lay persons who are trained as } \\
\text { facilitators may be less influenced by } \\
\text { previous experience than health } \\
\text { professionals, but they may not } \\
\text { possess the breadth of health } \\
\text { knowledge. Those who recruit, } \\
\text { select and train facilitators should be } \\
\text { aware of these issues. } \\
\text { Although facilitators may have a } \\
\text { health background, they may not } \\
\text { have behaviour change skills } \\
\text { required in psychosocial } \\
\text { interventions. Some may be } \\
\text { comfortable and confident in giving } \\
\text { information rather than using } \\
\text { strategies to induce behaviour } \\
\text { change. } \\
\text { Interventions that involve couples or } \\
\text { other family members may require } \\
\text { specific training to ensure that all } \\
\text { parties are fully engaged in the } \\
\text { sessions. Senn and Carey (2012) } \\
\text { reported that facilitating workshops } \\
\text { for men was more difficult than with } \\
\text { women. When men and women are } \\
\text { in the same workshop, group } \\
\text { interaction can present its own } \\
\text { problems. } \\
\text { A pilot phase, with real participants, } \\
\text { would have helped to identify these } \\
\text { some of these problems. It is } \\
\text { important for facilitators to reflect on }\end{array}$ \\
\hline
\end{tabular}




\begin{tabular}{|c|c|c|}
\hline $\begin{array}{l}\text { Values and attitudes } \\
\text { regarding principles } \\
\text { and beliefs } \\
\text { underpinning the } \\
\text { intervention }\end{array}$ & $\begin{array}{l}\text { of the intervention. } \\
\text { Some also found it hard to } \\
\text { keep to the structure of the } \\
\text { programme. } \\
\text { The co-facilitator in this study, } \\
\text { who was also a member of } \\
\text { the research team, monitored } \\
\text { the performance of facilitators } \\
\text { with regards to the fidelity of } \\
\text { the intervention. This was for } \\
\text { the purpose of recording what } \\
\text { happened and helping } \\
\text { facilitators to refrain from } \\
\text { deviating from the agreed } \\
\text { protocol. } \\
\\
\text { In this study, there was some } \\
\text { indication that sexual health } \\
\text { was an issue that some } \\
\text { facilitators did not feel } \\
\text { comfortable to discuss. This } \\
\text { may have been because they } \\
\text { did not have sufficient skills to } \\
\text { do so or that they had } \\
\text { particular views on sexual } \\
\text { issues that were different } \\
\text { from those embodied in the } \\
\text { intervention. }\end{array}$ & $\begin{array}{l}\text { their performance and to recognise } \\
\text { aspects of the intervention which } \\
\text { they find problematic and be } \\
\text { supported in order to improve their } \\
\text { skills and competence. Peer } \\
\text { supervision in the pre-training phase } \\
\text { would encourage facilitators to learn } \\
\text { from one another. } \\
\text { The purpose of monitoring and } \\
\text { supervising facilitators should be } \\
\text { both for recording what happened } \\
\text { and for ensuring that the intervention } \\
\text { is delivered as planned. A research } \\
\text { team member, not the co-facilitator, } \\
\text { should monitor the delivery. } \\
\text { Opportunities for debriefing (as } \\
\text { recommended by Senn and Carey, } \\
2012 \text { and Hoey et al., 2009)) should } \\
\text { be provided. This could help } \\
\text { facilitators to self-assess and to } \\
\text { receive feedback on their } \\
\text { performance. } \\
\text { It is well-known that beliefs and } \\
\text { attitudes can influence behaviour. } \\
\text { Facilitators' values, beliefs and } \\
\text { attitudes towards the intervention, } \\
\text { and specifically those issues that are } \\
\text { sensitive and controversial should be } \\
\text { examined at the beginning of the } \\
\text { project. Senn and Carey (2012) } \\
\text { reported that it was important that } \\
\text { facilitators be non-judgmental about } \\
\text { issues such as sexual behaviour. }\end{array}$ \\
\hline
\end{tabular}


Figure 1 Conceptual model of factors, relating to facilitators, influencing the delivery of psychosocial interventions in the context of a randomised controlled trials



